\title{
A-STAR: A Mobile Ad Hoc Routing Strategy for Metropolis Vehicular Communications
}

\author{
Boon-Chong Seet ${ }^{2}$, Genping Liu ${ }^{1}$, Bu-Sung Lee ${ }^{1}$, Chuan-Heng Foh ${ }^{1}$, \\ Kai-Juan Wong ${ }^{3}$, and Keok-Kee Lee ${ }^{1}$
}

1 Centre for Multimedia and Network Technology, Nanyang Technological University, Singapore. \{asgpliu, ebslee, aschfoh, askklee\}@ntu.edu.sg

2 Network Technology Research Centre, Nanyang Technological University,

Singapore, ebcseet@ntu.edu.sg

3 Institute for Computing Systems Architecture Informatics

University of Edinburgh, U.K., k.j.wong@sms .ed.ac.uk

\begin{abstract}
One of the major issues that affect the performance of Mobile Ad hoc NETworks (MANET) is routing. Recently, position-based routing for MANET is found to be a very promising routing strategy for inter-vehicular communication systems (IVCS). However, position-based routing for IVCS in a built-up city environment faces greater challenges because of potentially more uneven distribution of vehicular nodes, constrained mobility, and difficult signal reception due to radio obstacles such as high-rise buildings. This paper proposes a new position-based routing scheme called Anchor-based Street and Traffic Aware Routing (A-STAR), designed specifically for IVCS in a city environment. Unique to A-STAR is the usage of information on city bus routes to identify an anchor path with high connectivity for packet delivery. Along with a new recovery strategy for packets routed to a local maximum, the proposed protocol shows significant performance improvement in a comparative simulation study with other similar routing approaches.
\end{abstract}

\section{Introduction}

MANET is an autonomous system composed of mobile nodes communicating through wireless links in an environment without any fixed infrastructure support. Nodes in this network are self-organizing and rely on each other to relay messages to their correct destinations. As nodes are free to move randomly, the network topology may change rapidly and unpredictably. Thus, the routing protocol must be able to adapt and maintain routes in the face of changing network connectivity. Such networks are very useful in military and other tactical applications such as emergency rescue or exploration missions where an established (e.g. cellular) infrastructure is unavailable or unusable. Commercial applications are also likely where there is a need for ubiquitous communication services. Particularly in recent years, there is a growing commercial interest on the research and deployment of MANET technology for vehicular communications, e.g. FleetNet [1], VICS [2], CarNet 3 [3], etc. 
Existing MANET routing protocols work well in scenarios where nodes are uniformly distributed and moving freely in open space. However, these protocols do not work as well for IVCS in a city environment because of some additional inherent challenges. Generally, vehicular nodes are more unevenly distributed due to the fact that vehicles tend to concentrate more on some roads than others. Their constrained mobility by road patterns, along with more difficult signal reception in the presence of radio obstacles such as high-rise buildings, have contributed to greater fragility in the connectivity of the IVCS network, and the frequent formation of topology "holes", which could not be dealt with effectively by existing position-based routing protocols.

Recently, a project called BUSNet [4] was initiated to study the performance of MANET routing algorithms in the IVCS, based on a Metropolitan Grid model (M-Grid) [4][5]. It proposes using the regular network of buses to form a stable communication backbone for an otherwise fragile IVCS network. In [5], the performance of existing MANET routing protocols is found to be much lower in the M-Grid model than in the random waypoint model. This is because inter-node connectivity is much harder to establish with constrained mobility and obstacles in the M-Grid model.

For a large, metropolitan-scale IVCS network, the scalability of the routing protocol is very important. Position-based routing is known to be very scalable with respect to the size of the network. Thus, it is a good candidate for metropolitan-scale IVCS. However, applying position-based routing to IVCS may not be without any problems. An example is Greedy Perimeter Stateless Routing (GPSR) [6], one of the most well known position-based protocols in literature. It works best in a free open space scenario with evenly distributed nodes. But when applied to city scenarios [7][8], GPSR is found to suffer from several deficiencies, the details of which we will discuss in the next section.

This paper proposes a new position-based routing scheme called Anchorbased Street and Traffic Aware Routing (A-STAR), designed specifically for IVCS in a city environment. Unique to A-STAR is the usage of information on city bus routes to identify an anchor path with high connectivity for packet delivery. Along with a new recovery strategy for packets routed to a local maximum(to be explained in Section 2), the proposed protocol shows significant performance improvement in the M-Grid model. A-STAR is therefore proposed as a potential routing strategy for metropolis vehicular communications.

The remainder of the paper is organized as follows. Section 2 discusses with example the challenges faced by position-based routing in IVCS. Section 3 presents some works in literature related to this area. Section 4 describes the proposed A-STAR protocol. The mobility model and simulation setting are explained in Section 5. Performance results are presented in Section 6. Finally, the paper is concluded in Section 7. 


\section{Challenges of Position-Based Routing in IVCS}

The challenges of position-based routing in a city environment have been discussed thoroughly in [7][8]. An example is given here to illustrate some main problems if typical GPSR is deployed directly to IVCS. Figure 1 shows a partial city environment.

Suppose node $s$ wants to send a packet to node $d$. Greedy forwarding will fail in this case as there is no neighbor of $s$, which is nearer to $d$ than $s$ itself. Such a situation is what is commonly known as local maximum. Following the strategy in GPSR, the packet enters into perimeter-mode, using the right hand rule to travel through each node on the dotted route, including nodes $a, b$ and $c$. At $b$, it is found that $c$ is nearer to $d$ than $s$, at which the packet enters into perimeter-mode. Thus, the packet switches back to greedy mode at $b$, and then reaches its destination $d$ through $c$. It can be seen that this route is very long in terms of hop count. In fact, $s$ can reach $a$, and $a$ can reach $b$, both in one hop. This shows that the perimeter-mode which packet employs to recover from local maximum is very inefficient and time-consuming.

Another observation is that the packet can actually travel from $s$ to $d$ via a route that passes through $e$ and $f$ (shown as solid line), which is much shorter. However, this route is not exploited because the perimeter-mode of GPSR based on right hand rule is biased to a specific direction when selecting for the next hop.

It should be noted that in a city environment, the constrained mobility and frequently encountered obstacles can effectively force GPSR to run into perimeter-mode frequently. As a result, the performance of GPSR could deteriorate dramatically, and therefore may not be suitable for IVCS.

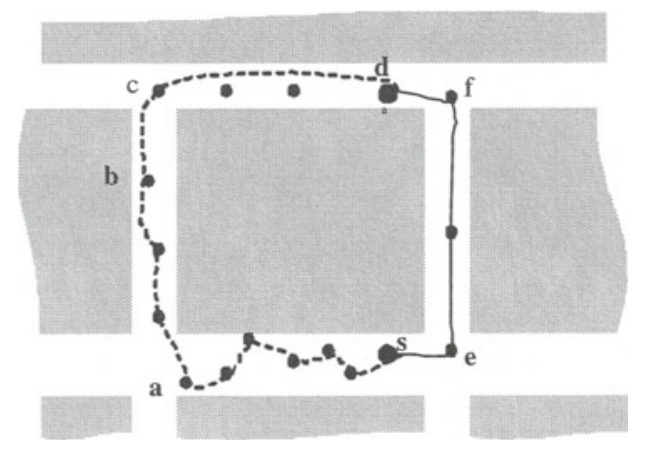

Fig. 1. Challenges of Position-Based Routing in IVCS 


\section{Related Work}

\subsection{Anchor-Based Routing}

Anchor-based routing is analogous to the source routing of DSR [10]. In anchorbased routing, the source node includes into each packet a route vector composed of a list of anchors or fixed geographic points, through which packets must pass. Between anchors, the greedy position-based routing is employed. Both Terminode Remote Routing (TRR) [9] and Geographic Source Routing (GSR) [7][8] are examples of algorithms that employ anchor-based routing to forward packets to remote destinations.

\subsection{Spatial Aware Routing}

In spatial aware routing, spatial information such as streets map of a city or a description of how several towns are connected by highways, is utilized to assist in making routing decisions. The spatial information reflects the underlying node distribution and topology of the network. Spatial aware routing is usually used in conjunction with anchor-based routing, such as in TRR and GSR where anchored paths are computed using the spatial information.

\section{Anchor-Based Street and Traffic Aware Routing (A-STAR)}

Considering the challenges faced in a city environment, a new position-based routing scheme called A-STAR is proposed. Similar to GSR, A-STAR adopts the anchor-based routing approach with street awareness. The term "street awareness" is preferred over "spatial awareness" to describe more precisely the use of street map information in our routing scheme for anchor path computation. That is, using the street map to compute the sequence of junctions (anchors) through which a packet must pass to reach its destination. But unlike GSR, ASTAR computes the anchor paths with traffic awareness. "Traffic" herein refers to vehicular traffic, including cars, buses, and other roadway vehicles.

It is observed that in a metropolitan area, some streets are wider and accommodate more vehicular traffic than others. These are the major streets, served by a regular fleet of city buses. Connectivity on such streets can be higher due to higher density of vehicular nodes and more stable due to regular presence of city buses. With this observation, weight can be assigned to each street based on the number of bus lines by which it is served, i.e. the more bus lines by which a street is served, the less weight it is assigned, and vice-versa. The street map in use by the vehicle is assumed to be loaded with bus route information. An anchor path can thus be computed using Dijkstra's least-weight path algorithm. For such a map with pre-configured information, it is called a statistically rated map.

While bus route information can provide a reasonable estimate of the expected vehicular traffic on each street, the traffic conditions in a city area can be 
Let $R$ be a node receiving a packet $p$ for destination $D$

Let $N$ be the set of one-hop neighbors of $R$

Let $A P$ represent the anchor path in the header of $p$

Let $L$ represent the number of hops $p$ has traversed

Let $L_{\max }$ represent the maximum hops $p$ is allowed to traverse

Let $L R$ represent the number of times $p$ has been recovered

Let $L R_{\max }$ represent the maximum number of times $p$ is allowed to be recovered If $(R=$ source $S$ of $p)$

Initialize $A P=$ null

Else If $\left(L \geq L_{\max }\right)$ or $\left(L R>L R_{\max }\right)$

Discard $p$

Return

If "out of service" information present in the header of $p$

Forward:

Update local map with the "out-of-service" information

If $(A P=$ null $)$

//anchor path initialization

Set $A P=$ least weight path from $R$ to $D$ with Dijkstra algorithm

If $(A P=$ null $)$

$/ /$ no anchor path exists, drop the packet

Discard $p$

Return

//compute the next hop $n$ along the anchor path

If ( $\exists n \in N: n$ resides on $A P$ and has shortest distance along $A P$ to $D$ )

Forward $p$ to $n$

Else // local maximum occurs

Mark the street where $n$ resides as "out of service" for time period $T$

Record the "out of service" information in the header of $p$

Set $A P=$ null

Goto Forward

Fig. 2. Pseudo code of A-STAR algorithm

quite dynamic at times. A better weight assignment scheme is therefore one that dynamically monitors and assigns weight to a street based on its latest traffic condition, which can provide higher quality of anchor computation. It could be envisaged that future IVCS would be able to monitor the city traffic condition and distribute such information to every vehicle connected to the IVCS network. This information could then be used to re-compute the weight of each street on the map, e.g. more vehicles, less weight assigned, and vice-versa. Such a map with re-configurable information is called a dynamically rated map.

\subsection{Local Recovery}

It has been shown that local recovery algorithm of GPSR using perimeter-mode is quite inefficient in a city area. Other recovery algorithms that rely on "right hand rule" such as face-1 or face-2 [11] also face a similar problem. GSR adopts 
a "switch back to greedy" approach for local recovery: when a packet reaches a local maximum along its anchor path, it switches back to greedy mode. This is not efficient at all as it has been shown that greedy forwarding does not perform well in a city environment.

Thus, a more efficient recovery strategy is proposed for A-STAR: a new anchor path is computed from the local maximum to which the packet is routed. The packet is salvaged by traversing the new anchor path. To prevent other packets from traversing through the same void area, the street at which local maximum occurred is marked as "out of service" temporarily, and this information is distributed to the network by piggybacking them onto the packets to be recovered. Nodes receiving these packets update their local map with the "out of service" information prior to making their forwarding decision. The "out of service" streets are not used for anchor computation or re-computation during the "out of service" duration and they resume "operational" after the time out duration. A maximum threshold value $\left(L R_{\max }\right)$ is also defined to limit the number of times a packet can be recovered to prevent the perpetual sending of outdated data and bandwidth wastage. Figure 2 presents the pseudo code of the A-STAR algorithm.

\section{$5 \quad$ Mobility Model and Simulation Setting}

\subsection{M-Grid Mobility Model}

Mobility model describes the movement of nodes in a certain environment. In this paper, the M-Grid mobility model [4][5] is used to describe the movement of vehicular nodes in a city area. M-Grid is a variant of the Manhattan model [12], which models the vehicular movement in a typical metropolis where streets are set out on a grid pattern. Key features which distinguish the M-Grid from Manhattan model, include:

- Node heterogeneity: Buses and cars are two types of vehicular nodes modeled in our M-Grid. Buses, which only travel along the bus routes, show higher regularity and lower mobility than cars. For the M-Grid in Figure 3, the bus routes are represented by bold lines in gray. It shows three loop lines (or service numbers), plying the streets in various parts of the city. Each line is bi-directional with buses running clockwise and anti-clockwise.

- Preferential movements: It is observed that in real life, some streets would attract more vehicles than others. More often than not, these are the main streets, which are bustling with people and therefore served by buses. In MGrid, when a car reaches a junction, it would choose to move into another street with some preference. Given the observation above, the car at the junction shall give greater preference to a street which is on a bus route than one which is not.

- Radio obstacles: The blocking of signal transmissions by objects such as high-rise buildings in the city has been modeled in M-Grid. As Figure 4 shows, the gray areas represent obstacles, which are non-penetrable by the 


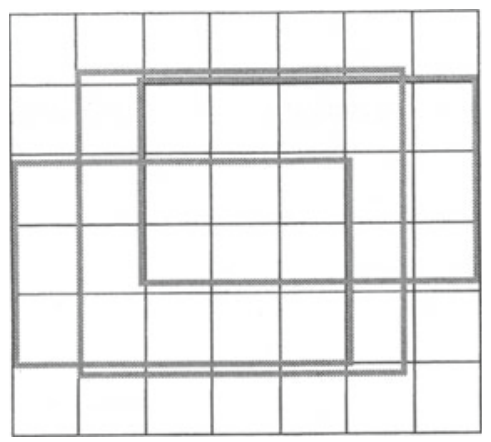

Fig. 3. M-Grid with bus routes

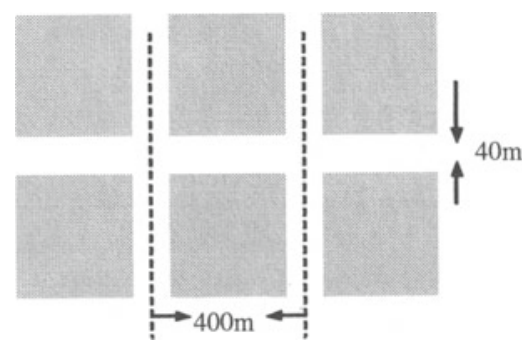

Fig. 4. M-Grid with obstacles

signals. Thus, for a node pair to communicate directly, they must have a "line-of-sight" to each other, in addition to being in range of one another.

\subsection{Simulation Setting}

Performance of A-STAR and other related protocols are evaluated using the ns2 [13] simulator. Four protocols are implemented, namely: i) GPSR, ii) GSR, iii) A-STAR-SR, and iv) A-STAR-DR. Protocol iii and iv refer to the proposed A-STAR with statistically rated and dynamically rated maps respectively. The presence of an information system that provides location service (e.g. [14] offering information about the position of other network users) and current road traffic state is assumed. Table 1 summarizes the parametric settings used in our simulation.

Note that the number of vehicles (nodes) is varied to reflect different vehicle densities under which the performance of each protocol is evaluated. However, throughout the evaluation, the number of buses is a constant, with only the car density varying. Inter-bus distance is approximately 1 kilometer for each line in the same direction. With three bus lines for the M-Grid shown in Figure 3, a total of 37 buses will be running in the city: two with 12 buses, one with 13 buses. Moreover, cars at the junction would move into a street which is on a bus route with a probability three times that of which is not(to effect the preferential 
movements in the M-Grid as mentioned in Section 5.1). Speed limit of buses and cars are 50 and $70 \mathrm{~km} / \mathrm{h}$ respectively.

Performance result for each simulated vehicle density (node number) is the average of five simulation runs. The key metrics of interest are:

- Packet delivery ratio: the ratio of packets delivered to the destinations to those generated by the sources.

- End-to-end delay: the average time it takes for a packet to traverse the network from its source to destination.

Results of the control overhead is not presented here because the overhead messages are predominantly beacon messages transmitted periodically by nodes to build up their neighbors' location information, the amount of which are the same for all position-based routing protocols considered in this study.

Table 1. Simulation Setting

\begin{tabular}{|c|c|}
\hline Parameter & Setting \\
\hline Mobility model & M-Grid \\
\hline Traffic model & 20 CBR connections \\
\hline Packet sending rate & 4 packets $/$ second \\
\hline Data packet size & 64 bytes \\
\hline Transmission range & 350 meters \\
\hline Map size & $2800 \times 2400 \mathrm{~m} 2$ ( $7 \times$ grid $)$ \\
\hline Node number & 200 to 500, in steps of 50 \\
\hline Simulation time & 500 seconds \\
\hline MAC protocol & IEEE 802.11 DCF \\
\hline
\end{tabular}

\section{Simulation Results and Analysis}

Recall that A-STAR differs from GSR and GPSR in two main aspects. Firstly, ASTAR incorporates traffic awareness by using statistically rated and dynamically rated maps. Secondly, A-STAR employs a new local recovery strategy that is more suitable for a city environment than the greedy approach of GSR, or the perimeter-mode of GPSR.

To investigate impacts of each aspect on the routing performance, protocols are evaluated initially without local recovery, and later with local recovery. Without local recovery, a packet is simply dropped when it encounters a local maximum. Figures 5 and 6 show the protocols performance (with $95 \%$ confidence intervals) without and with local recovery, respectively.

In Figure 5(a), it is observed that more packets are delivered as node number increases. This is expected since more nodes increases the probability of connectivity, which in turn reduces the number of packets dropped due to local 


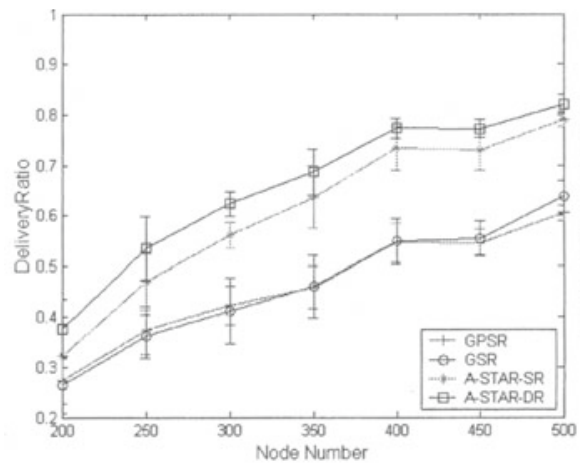

(a)Packet Delivery Ratio

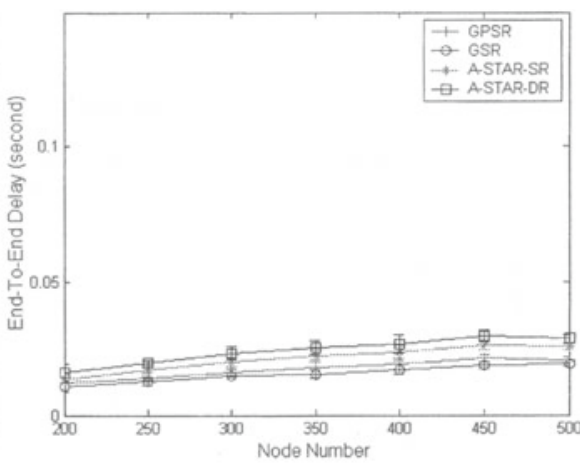

(b) End-to-End Delay

Fig. 5. Performance without local recovery

maximum. It is also observed that GSR did not show a better performance than GPSR, possibly because the grid layout of streets did not pose as much problem to GPSR as did one with fork junctions in [8]. With traffic awareness, A-STAR shows the best performance because it can select paths with higher connectivity for packet delivery. As much as $40 \%$ more packets are delivered by A-STAR, compared to GSR. Between A-STAR-SR and A-STAR-DR, the latter performs better by using more precise vehicular traffic information. Figure 5(b) shows the result of end-to-end delay. Generally, no significant difference is observed between the protocols. A-STAR, however, shows slightly higher delay that may be attributed to possibly longer, but higher connectivity paths used for packet delivery.

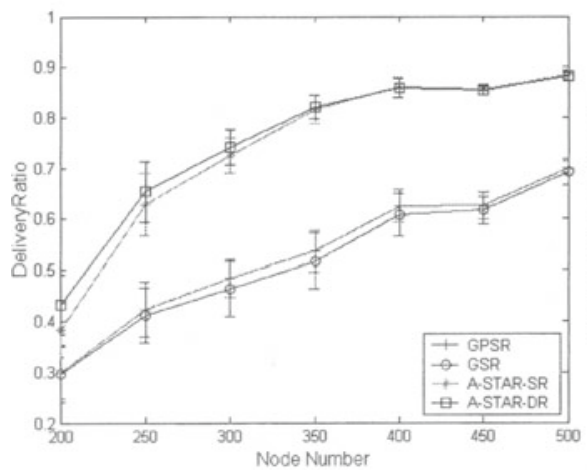

(a)Packet Delivery Ratio

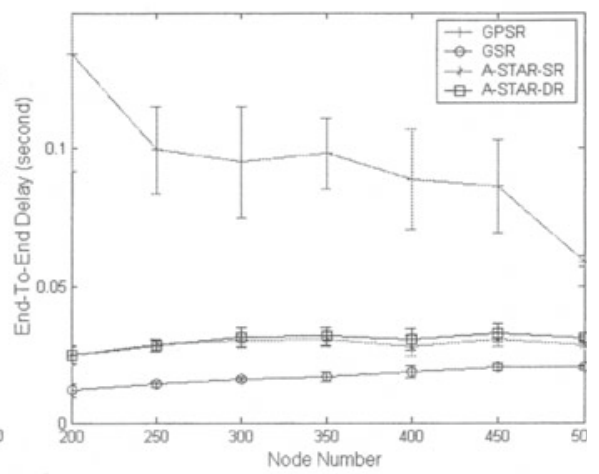

(b) End-to-End Delay

Fig. 6. Performance with local recovery 


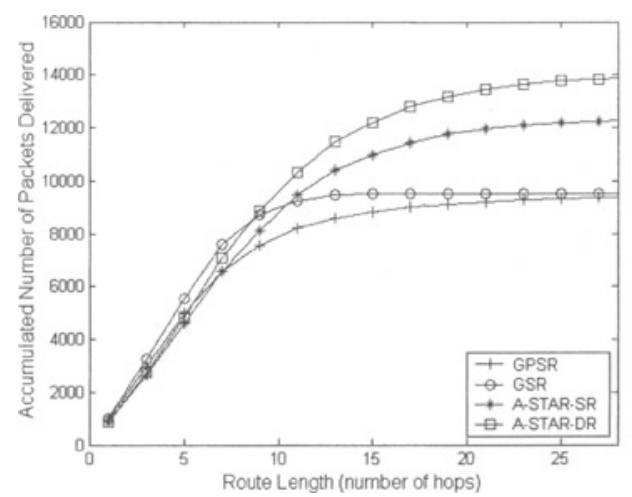

Fig. 7. Route length distribution (for 200 nodes)

With local recovery, packets that encounter local maximum can be rerouted and delivered instead of being dropped. Thus, more packets are delivered by each protocol as shown in Figure 6(a). The increase in packets delivered is more significant at lower node number where local maximum is encountered more frequently. For example, with local recovery, A-STAR-DR delivers $20 \%$ more packets at 250 nodes, while only $6 \%$ more at 400 nodes. It is also observed that local recovery allows A-STAR-SR to narrow its performance gap with A-STARDR. GSR and GPSR show improvement in packet delivery of not more than $15 \%$ with local recovery, which suggests that their recovery strategies may not be very effective in a city environment.

Figure 6(b) shows the corresponding result for end-to-end delay. A key observation is that GPSR with local recovery incurs significantly higher end-to-end delay. This is because of frequent attempts by GPSR to salvage packets from local maximum via perimeter-mode, which is generally inefficient and causes congestion especially at lower node number. Delay of A-STAR is lower than GPSR, but seemingly higher than GSR, once again at lower node number. A close analysis of its route length distribution in Figure 7 suggests that the higher delay is likely an artifact due to successful delivery of more long-distance packets that are otherwise dropped without local recovery. These packets inevitably have longer traversal time and thus contribute to a higher average end-to-end delay.

\section{Conclusion}

In this paper, a new position-based routing protocol A-STAR is proposed for metropolis vehicular communications. A-STAR features the novel use of city bus route information to identify anchor paths of higher connectivity so that more packets can be delivered to their destinations successfully. In our comparative simulation study with other position-based routing schemes, A-STAR demonstrates excellent improvement in packet delivery while maintaining reasonable end-to-end delay. As future work, the traffic awareness in A-STAR shall 
be extended to include data traffic to provide vehicular nodes with higher performance paths in terms of connectivity as well as delay. Another area that shall be looked into is how information on bus schedules, in addition to bus routes, can be utilized to further optimize the performance of our protocol.

\section{References}

1. W. Franz, R. Eberhardt, and T. Luckenbach, "FleetNet - Internet on the Road", Proc. 8th World Congress on Intelligent Transportation Systems, Sydney, Australia, Oct. 2001.

2. S. Yamada. "The Strategy and Deployment Plan for VICS", IEEE Communications, Vol. 34, No. 10, pp.94-97, 1996

3. R. Morris, J. Jannotti, F. Kaashoek, J. Li, and D. Decouto, "CarNet: A Scalable Ad Hoc Wireless Network System", Proc. 9th ACM SIGOPS European Workshop, Sept. 2000.

4. K. J. Wong, B. S. Lee, B. C. Seet, G. Liu, and L. Zhu, "BUSNet: Model and Usage of Regular Traffic Patterns in Mobile Ad Hoc Networks for Inter-Vehicular Communications", Proc. ICT 2003, Thailand, April, 2003

5. B. S. Lee, K. J. Wong, B. C. Seet, L. Zhu, and G. Liu, "Performance of Mobile Ad Hoc Network in Constrained Mobility Pattern", Proc. International Conference on Wireless Networks (ICWN'03), Las Vegas, USA, Jun. 2003.

6. B. Karp and H. T. Kung, "GPSR: Greedy Perimeter Stateless Routing for Wireless Networks", Proc. ACM/IEEE MobiCom, Boston, USA, Aug. 2000.

7. C. Lochert, H. Hartenstein, J. Tian, H. Füßler, D. Herrmann, and M. Mauve, "A Routing Strategy for Vehicular Ad Hoc Networks in City Environments", Proc. IEEE Intelligent Vehicles Symposium (IV2003), Ohio, USA, Jun. 2003.

8. J. Tian, I. Stepanov, and K. Rothermel, "Spatial Aware Geographic Forwarding for Mobile Ad Hoc Networks", Proc. MobiHoc, Lausanne, Switzerland, Jun. 2002.

9. L. Blazevic, S. Giordano, and J. Y. Le Boudec. "Self-Organizing Wide-Area Routing", Proc. SCI 2000/ISAS 2000, Orlando, USA, Jul. 2000.

10. D. B. Johnson and D. A. Maltz, "Dynamic Source Routing Protocol for Mobile Ad Hoc Networks", Mobile Computing, T. Imielinski and H. Korth, Eds., Kluwer, 1996, pp. 153-81.

11. P. Bose, P. Morin, I. Stojmenovic, and J. Urrutia, "Routing with Guaranteed Delivery in Ad Hoc Wireless Networks", Proc. 3rd ACM International Workshop on Discrete Algorithms and Methods for Mobile Computing and Communications (DIALM'99), Seattle, USA, Aug. 1999.

12. A. Kamat and R. Prakash. "Effects of Link Stability and Directionality of Motion on Routing Algorithms in MANETs". Proc. IEEE International Conference on Computer Communications and Networks (ICCCN), Las Vegas, USA, Oct 2000.

13. Network Simulator (ns-2), http://www.isi.edu/nsnam/ns/

14. M. Kasemann, H Fußler. Hartenstein, and M. Mauve. A Reactive Location Service for Mobile Ad Hoc Networks. Technical Report TR-02-014, Department of Computer Science, University of Mannheim, Nov. 2002. 